\title{
ACUTE CEREBELLITIS CAUSED BY HERPES
} SIMPLEX VIRUS

Clin Biomed Res. 2019;39(1):104-105

1 Serviço de Radiologia, Hospital de Clínicas de Porto Alegre (HCPA). Porto Alegre, RS, Brasil.

2 Serviço de Neurologia, Hospital de Clínicas de Porto Alegre (HCPA). Porto Alegre, RS, Brasil.

3 Serviço de Controle de Infecção Hospitalar, Hospital de Clínicas de Porto Alegre (HCPA). Porto Alegre, RS, Brasil.

4 Departamento de Medicina Interna, Universidade Federal do Rio Grande do Sul (UFRGS). Porto Alegre, RS, Brasil.

Corresponding author: Lillian Gonçalves Campos cglillian@yahoo.com Serviço de Radiologia, Hospital de Clínicas de Porto Alegre (HCPA) Rua Ramiro Barcelos, 2350. 90035-903, Porto Alegre, RS, Brasil.

\author{
Lillian Gonçalves Campos ${ }^{1}$, Roberto Rossato², \\ Rodrigo Pires dos Santos ${ }^{3}$, Juliana Avila Duarte ${ }^{1}$, Leonardo Vedolin ${ }^{1,4}$
}

\section{CASE PRESENTATION}

A 29 year-old woman presented to the emergency department with gait imbalance and dysarthria. At admission, neurological examination revealed normal cognition, ataxia, dysarthria, dysmetria on both sides of the body, bilateral vertical nystagmus and loss of the lateral eye movement. Blood examination showed an increase in white blood cell (WBC) count and demonstrated erythrocyte sedimentation rate of $18 \mathrm{~mm} / \mathrm{h}$. Examination of cerebrospinal fluid (CSF) revealed protein concentration of $166 \mathrm{mg} / \mathrm{dL}$, glucose concentration of $56 \mathrm{mg} / \mathrm{dL}$, and pleocytosis. Serum glucose concentration was $126 \mathrm{mg} / \mathrm{dL}$. The patient had no history of immunosuppression or any comorbidity and anti-HIV test was negative.

Neurological evaluation included a head computed tomography (CT) scan with normal findings. Magnetic resonance imaging (MRI) of the brain revealed increased bilateral signal intensity in the cerebellum on fluid-attenuated inversion recovery images (FLAIR)/T2, without contrast enhancement, suggesting an inflammatory process confined to the cerebellum (Figures 1 and 2). Furthermore, the cerebellar cortex appeared swollen, a finding consistent with diffuse cerebellitis. There were no changes in the brainstem. Initially, the possibility of bacterial rhombencephalitis caused by Listeria monocytogenes was considered, as it is the most common cause of rhombencephalitis.

After a few days of antibiotic therapy (ceftriaxone and ampicillin), polymerase chain reaction (PCR) test of the CSF was positive for herpes simplex virus $1 / 2$ (HSV). Bacterial culture of CSF samples showed no growth, and the results of Gram staining of CSF were negative. Anti-Listeria antibody was also negative, and ampicillin was discontinued. CSF PCR analysis for other herpes viruses (varicella-zoster virus, Epstein-Barr virus, cytomegalovirus, and human herpesvirus types 6-8) and enteroviruses were also negative.

Treatment with acyclovir ( $50 \mathrm{mg} / \mathrm{kg} /$ day) for 21 days improved symptoms. One month after the first MRI, a significant reduction in imaging abnormalities was detected (Figure 3).

\section{DISCUSSION}

Acute cerebellitis remains an unclear clinical entity that has been associated with multiple etiologies including viruses (e.g., varicella zoster virus (VZV), measles, mumps, rubella, Epstein-Barr virus (EBV), cytomegalovirus, herpes simplex virus, parainfluenza virus, enterovirus and coxsackie virus) and bacteria (e.g., Salmonella typhi, Borrelia burgdorferi, Coxiella burnetii, Bordetella pertussis, and Mycoplasma pneumoniae) ${ }^{1}$. The pediatric population is more frequently involved, with fewer cases described in adults ${ }^{1}$. Acute cerebellitis is a neurological condition characterized by mild or high-grade fever, nystagmus, tremor, truncal ataxia, dysarthria, headache, and altered mental state ${ }^{2}$. Hydrocephalus and tonsillar herniation are most likely to be complications of the acute phase and may require neurosurgical intervention to prevent death ${ }^{2}$.

HSV encephalitis has a predilection for the temporal and frontal lobes, but occasionally affects the brainstem and less commonly only the cerebellum ${ }^{3-5}$. 


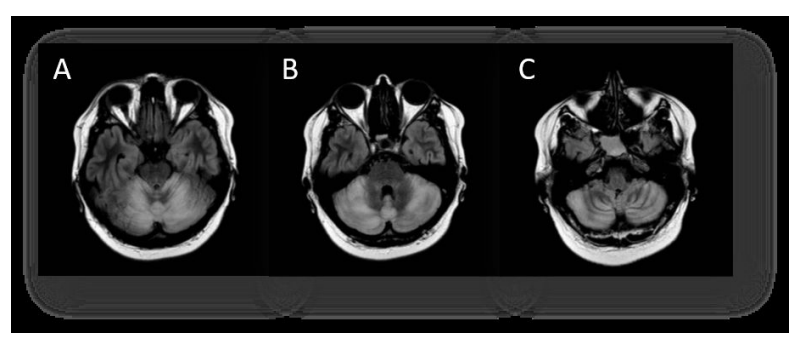

Figure 1: Imaging findings in acute cerebellitis associated with herpes simplex virus before and after treatment. Initial brain magnetic resonance imaging demonstrates symmetrical increase signal intensity on fluid-attenuated inversion recovery sequences (FLAIR) in the cerebellar hemispheres and vermis, especially in the cerebellum cortex that appeared swollen (A, B and C).

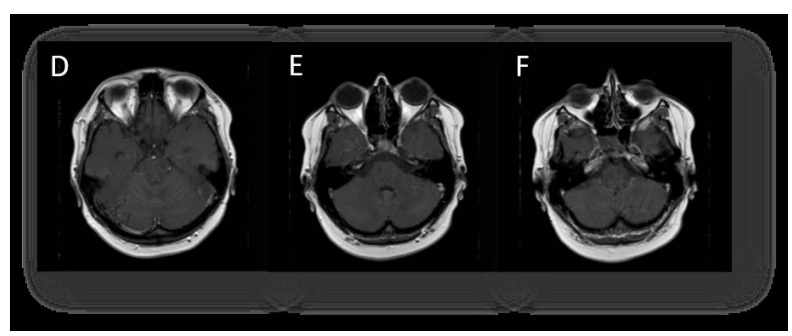

Figure 2: There was no contrast enhancement after intravenous gadolinium administration (D, E and $F$ ) and no restriction on diffusion (not shown).

When there is exclusive involvement of the cerebellum, bilateral diffuse hemispheric abnormalities represent the most common imaging presentation ${ }^{4}$. Sometimes the changes can be restricted to one hemisphere,

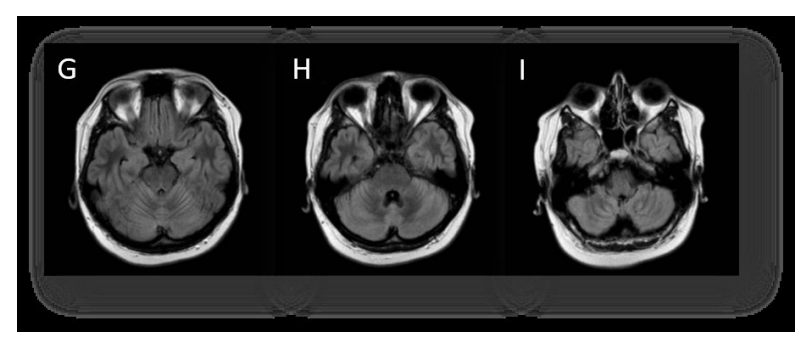

Figure 3: After the treatment was completed and symptoms improved, there was a significant regression of the areas of hyperintensity on fluid-attenuated inversion recovery images ( $\mathrm{G}, \mathrm{H}$ and $\mathrm{I})$.

vermis or peduncles ${ }^{4}$. MRI is more sensitive to assess the brainstem and cerebellum ${ }^{4}$. There are few cases of HSV acute cerebellitis reported in the literature, in the absence of cerebral or brainstem lesions.

Differential diagnosis of acute cerebellitis includes infectious agents (e.g., viruses and bacteria), acute disseminated encephalomyelitis, Lhermitte-Duclos disease, diffusely infiltrating glioma or lymphoma, vasculitis, ischemic stroke, autoimmune diseases, paraneoplastic syndromes and drug-related inflammatory processes (e.g., drug intoxication) ${ }^{4}$. Imaging studies can be a very important tool in the distinction of these processes, as there are overlapping clinical and laboratory findings.

\section{Conflicts of Interest}

The authors declare no conflicts of interest.

\section{REFERENCES}

1. Patel $\mathrm{P}$, Rayamajhi S, Tokala $\mathrm{H}$, Laird-Fick H. An unusual cause of altered mental status in elderly. Acute cerebellitis: a case report and review. Case Rep Med. 2013;2013:1-3. http:// dx.doi.org/10.1155/2013/653925. PMid:24382966.

2. Ciardi M, Giacchetti G, Fedele CG Tenorio A, Brandi A, Libertone R, et al. Acute cerebellitis caused by herpes simplex virus type 1. Clin Infect Dis. 2003;36(3):50-4. http://dx.doi. org/10.1086/345781. PMid:12539091.
3. Livorsi D, Anderson E, Qureshi S Howard M, Wang YF, Franco-Paredes C. Brainstem encephalitis: an unusual presentation of herpes simplex virus infection. J Neurol. 2010;257(9):14327. http://dx.doi.org/10.1007/s00415010-5600-x. PMid:20495814.

4. Bruecker $Y$, Claus F, Demaerel P, Ballaux F, Sciot R, Lagae L, et al. MRI findings in acute cerebellitis. Eur Radiol. 2004;14(8):1478-83. http:// dx.doi.org/10.1007/s00330-0042247-y. PMid:14968261.
5. Miura S, Kurita T, Noda K, Ayabe M, Aizawa H, Taniwaki T. Symmetrical brainstem encephalitis caused by herpes simplex virus. J Clin Neurosci. 2009;16(4):58990. http://dx.doi.org/10.1016/j. jocn.2008.06.005. PMid:19201192.

Received: Oct 8, 2018 Accepted: Mar 13, 2019 